\title{
Joint Transform Correlation \\ in Security Applications
}

\author{
Miloš Klíma*, Jiří Rott*, Thomas Naughton+, John Keating+ \\ ${ }^{\star}$ Faculty of Electrical Engineering, Czech Technical University, \\ Technická 2, 16627 Praha 6, Czech Republic, Email: klima@feld.cvut.cz \\ + Dept. of Computer Science, Maynooth College, \\ Maynooth, Co.Kildare, Republic of Ireland
}

Abstract - The Joint Transform Correlation (JTC) technique is the one of the most frequently applied methods in the field of optical classification and identification systems. Nowadays there are a lot of different modifications [1] that have been tested and verified. Because of extremely high computational throughput the JTC has been implemented in many special purpose (security) applications. This paper deals with an implementation of the JTC for two basic classes of objects - fingerprints and faces. In the first part the sensitivity to rotation and zooming is studied. Consequently in the second part two types of thresholding in the spectral domain are tested in order to improve performance of the system. All procedures are simulated on a computer first and then the real optical FT setup is employed. The results are compared and discussed.

\section{INTRODUCTION TO THE JTC}

The JTC identification method is based upon a twofold consequent application of a Fourier transform with a non-linearity (usually quadratic) in the spectral domain for an evaluation of the true 2D correlation of two images. The fundamental JTC setup is sketched in Fig. 1. The laser beam is expanded and then passes through the transparency assembled from two parts $r(x, y)$ as a reference and $s(x, y)$ as a test placed side by side. The first Fourier transform lens Ll creates the joint Fourier transform of the original image $r(x, y)+s(x, y)$ in its rear focal plane. This operation can be described by the well known equation (1)

$$
\begin{aligned}
& I(u, v)=|F T[(r(x-a, y)+s(x+a, y))]|^{2}= \\
& =|R(u, v)+S(u, v)|^{2}=R(u, v) R^{*}(u, v)+S(u, v) S^{*}(u, v)+ \\
& +R(u, v) S^{*}(u, v) \exp (i 2 \pi u a)+S(u, v) R^{*}(u, v) \exp (-i 2 \pi u a) \\
& \text { where }
\end{aligned}
$$

$I(u, v)$ - intensity distribution in the Fourier plane,

a - off-axis shift.
The created spectrum undergoes a quadratic nonlinear transformation and the squared spectrum is subjected to the second inverse Fourier transform by the second transformation lens L2. The optical intensity field in the rear focal plane of lens $\mathrm{L} 2$ can be described by equation (2). The symmetrical $2 \mathrm{D}$ correlation (autocorrelation and crosscorrelation) peaks appear there as shown in equation (2) and they are located:

$$
\begin{array}{ll}
\text { at the point } 0 & r(x, y) \otimes r(x, y)+s(x, y) \otimes s(x, y) \\
\text { at the point }+2 \mathrm{a} & s(x, y) \otimes r(x, y) \\
\text { at the point -2a } & r(x, y) \otimes s(x, y)
\end{array}
$$
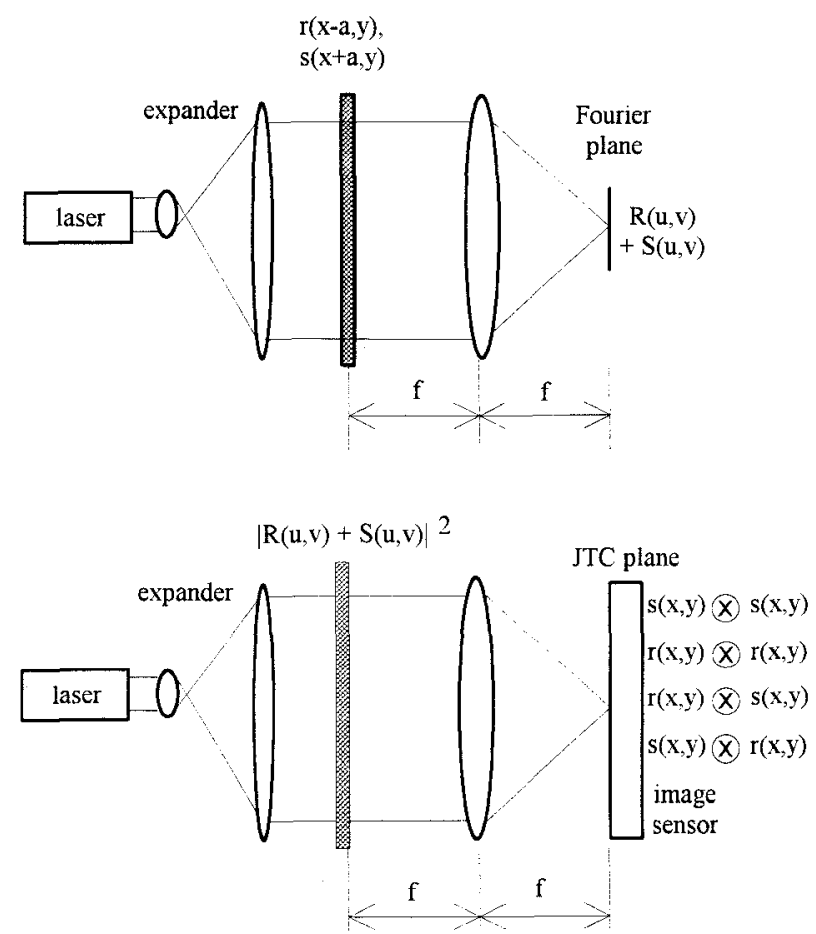

Fig. 1: JTC setup 


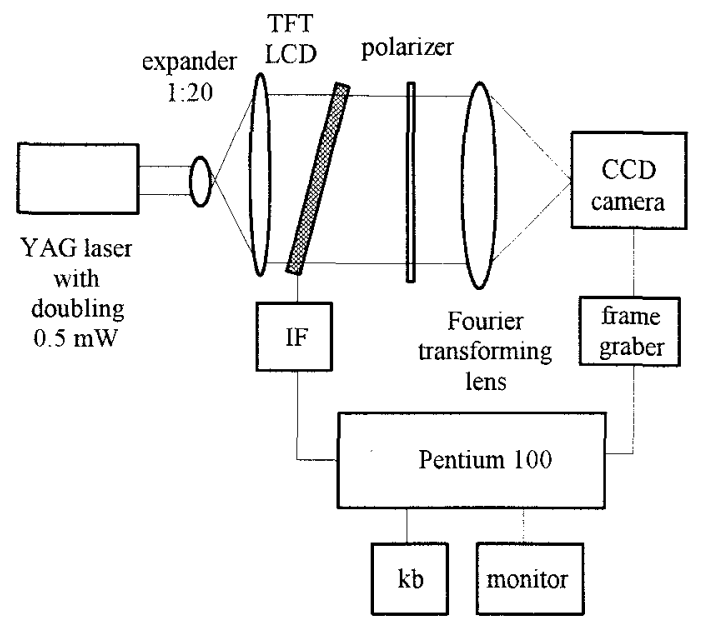

Fig.2: Experimental JTC setup

The experimental setup is usually split into two consequent steps as shown in Fig.2. For the first transparency a standard 2D LCD panel is used and both images $\mathrm{r}+\mathrm{s}$ are displayed on it. The joint spectrum is picked up by a TV camera (2D array sensor) and the detection process itself provides the required quadratic non-linearity. After that the detected joint spectrum is displayed again on a LCD panel to perform the inverse Fourier transform and both Fourier transforms are provided by the same lens $\mathrm{L}$.

\section{DESCRIPTION OF ANALYSIS}

In the following paragraphs we would like to present the results of our tests of JTC performance. The first part is devoted to the most critical points of the JTC - the sensitivity to rotation and dilation (zooming) of a test image. As the test image we have used two different patterns which are very interesting from a security point of view - fingerprints and faces In both cases it is expected some image preprocessing will be required before it is subjected to the JTC algorithm

The nature of fingerprints is more or less a periodic binary structure and we have applied a simple thresholding on the input images in order to eliminate some residual noise between lamels. Fig. 3 . shows a set of input test pictures after preprocessing

A face image is a typical grey level image. The significant value for the mean brightness in image resulted in a poor JTC performance. Therefore we have applied an edge detection convolution filtering and a thresholding on the images. This way we have got the binary image similar to a fingerprint - see Fig.4. Nevertheless this procedure preserves significant identification details such as eyes, eyebrows, mouth, nose, ears and hair form etc. In all cases diagrams of the correlation peak amplitude are presented as functions of a particular parameter - rotation or scaling.

The second part of our experiments deals with a thresholding in a spectral domain. This part has not been well theoretically described yet because of operator non-linearity but there has been promising results published. We have implemented two different types of thresholding constant and exponential. Again as in the first part diagrams of correlation peak amplitude are presented as functions of a thresholding level

In both parts the exact calculations have been done by a mathematical simulation on a computer with the mathematical software package MATLAB. In order to verify the mathematical simulation the above mentioned experimental optical Fourier transform setup has been used for the inverse (second) Fourier transform. The results of the simulation and optical transform are presented and discussed.
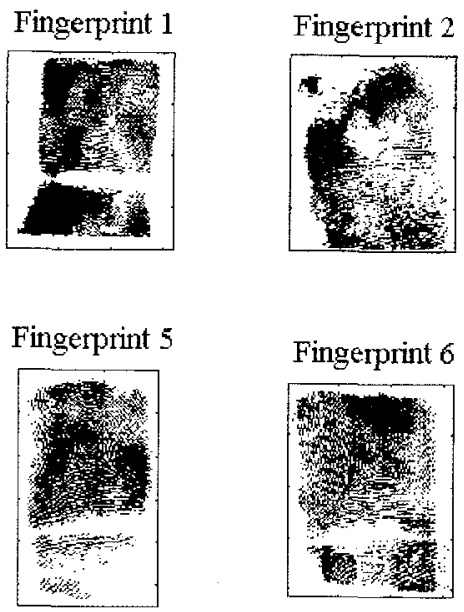

Fig. 3: Preprocessed test images - fingerprints
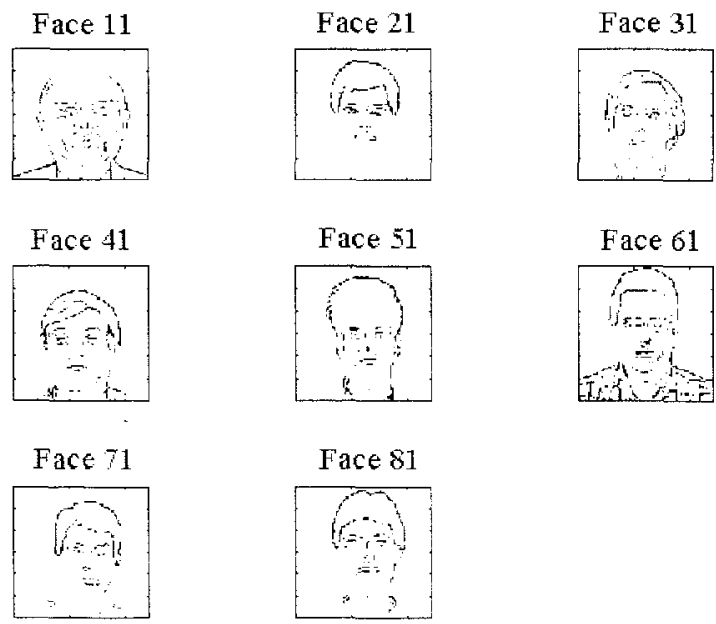

Fig 4: Preprocessed test images - faces
Fingerprint 3

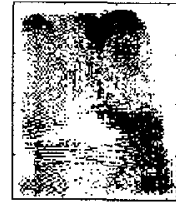

Fingerprint 7

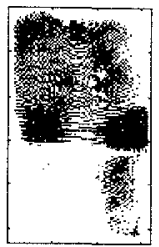




\section{ROTATION}

In the case of fingerprints the rotation is a fundamental problem. The dependence of the correlation (autocorrelation) peak amplitude on rotation is shown in Fig.5. The amplitude of correlation peak is measured in arbitrary units. The diagram has been calculated with fingerprint No.6 used as both a reference and a test. For comparison the other fingerprints have been correlated with No.6 and the results are shown in the centre. It is apparent that the detection of similarity is useless out of the range from 5 to 5 degrees - no difference in the amplitude of correlation peaks (ratio 1:1). The correlation field at the output of the real optical setup is demonstrated on Fig. 6.

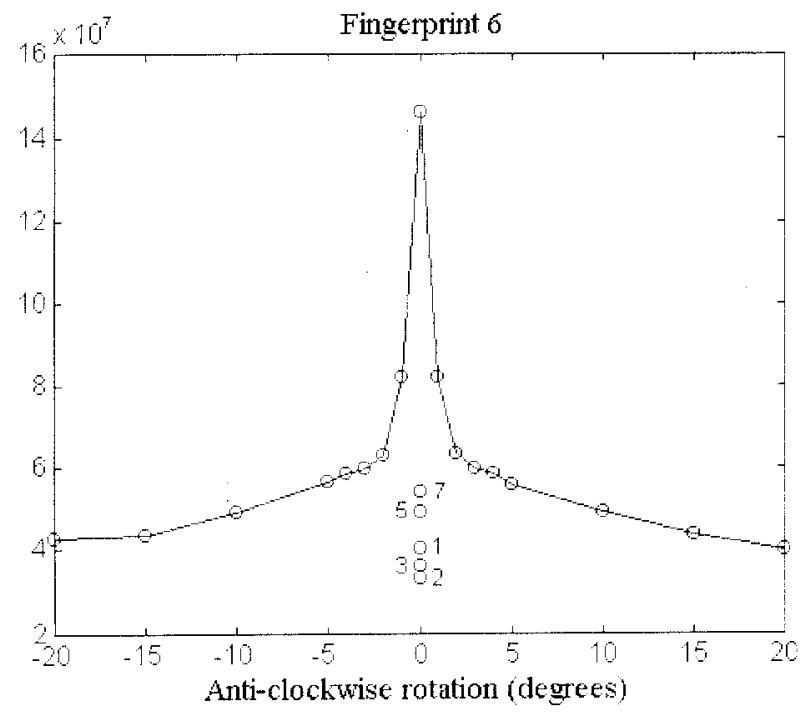

Fig.5: Correlation peak vs. rotation - fingerprint No.6

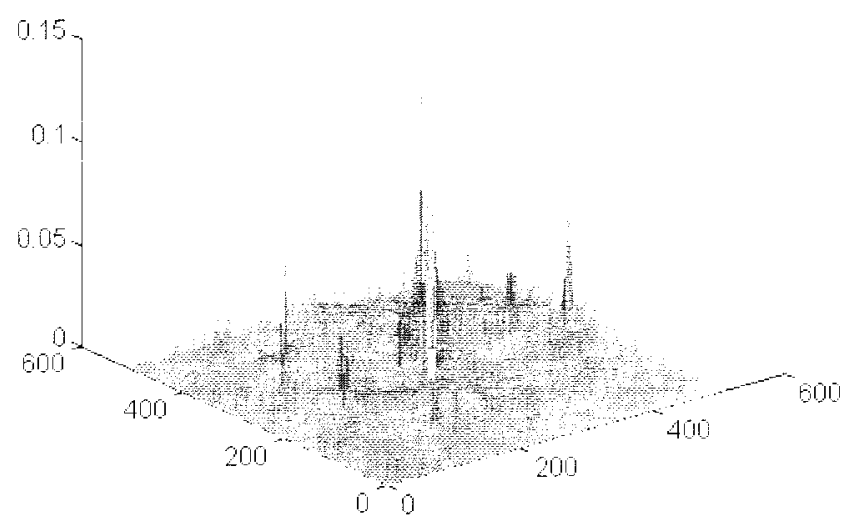

Fig.6: Real JTC output - fingerprint No.6
The fundamental image characteristics of human faces are slightly different (non-periodic). Face No.3 has been used as a reference. The useful detection range is extended to approx. -10 to 10 degrees with the ratio 1:1, see Fig. 7 .

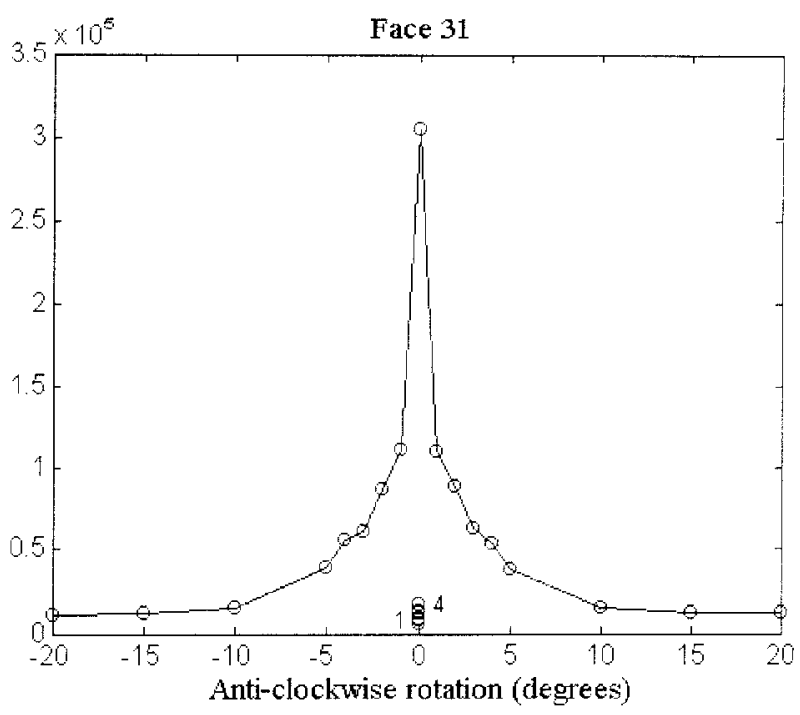

Fig 7: Correlation peak vs. rotation - face No.31

\section{ZOOMING (DILATION)}

Dilation is the other possible distortion of an input picture. The relevant dependencies for fingerprints and faces are sketched in Fig. 8. and Fig. 9 respectively. These dependencies are naturally non-symmetrical and another local extreme has appeared for faces.

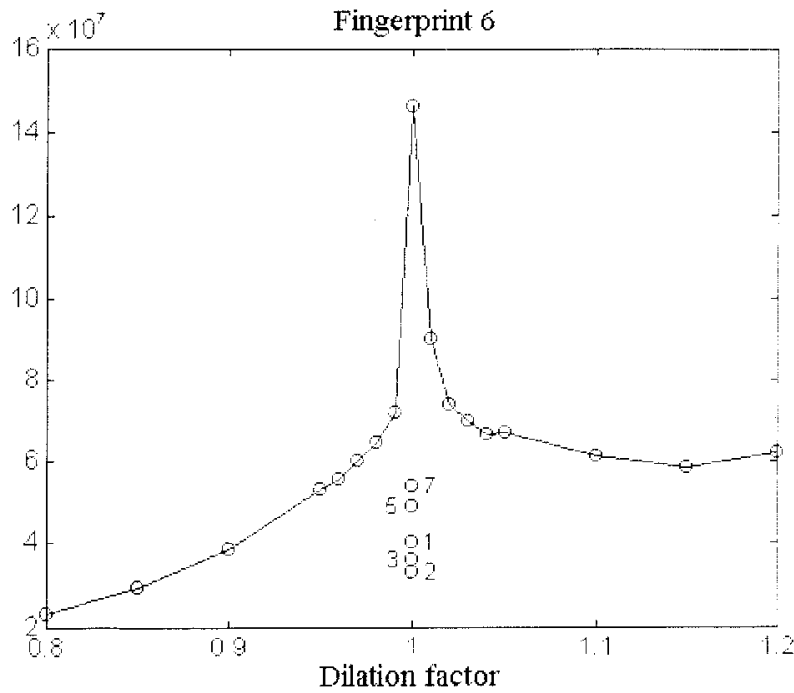

Fig. 8: Correlation peak vs. dilation - fingerprint No.6 


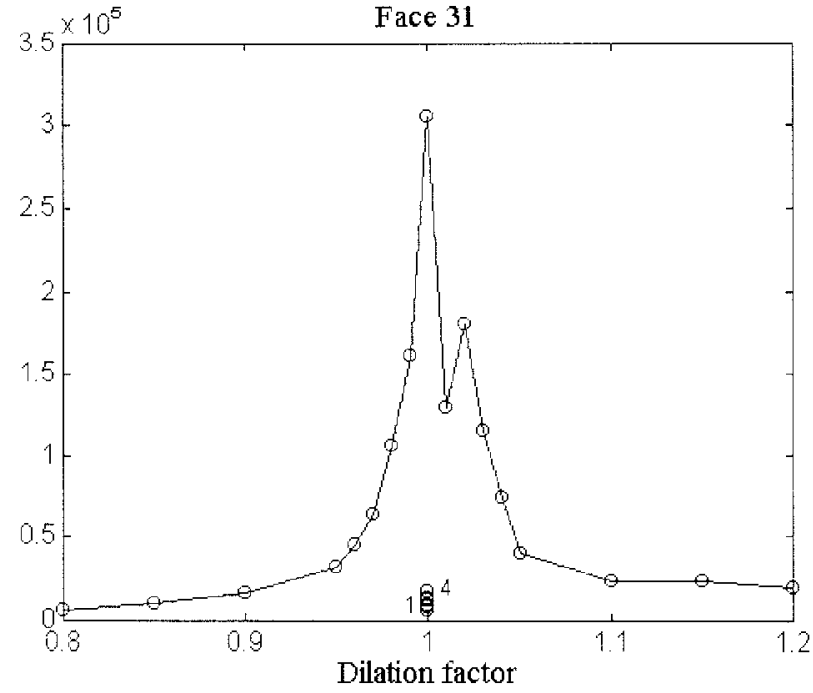

Fig.9: Correlation peak vs. dilation - face No.31

\section{UNIFORM THRESHOLDING IN THE SPECTRAL PLANE}

Another operation we have applied is a binarization in the spectral plane - a constant thresholding (independent to spatial frequencies). This is a non-linear operation with significant consequences in the correlation plane. In Fig. 10 an example of the thresholded spectrum of fingerprints is shown. In order to maximize the amplitude of the correlation peak the dependence of the correlation peak amplitude on the threshold level has been drawn - see Fig. 1 1, 12. The thresholding level is calculated relative to the amplitude of the DC component in the spectral plane. In both cases fingerprints Fig. 11 and faces Fig. 12 - we can localize some optimum thresholding levels at about $10^{-2}$ to $10^{-4}$. The thresholded spectrum for fingerprints is shown in Fig. 13

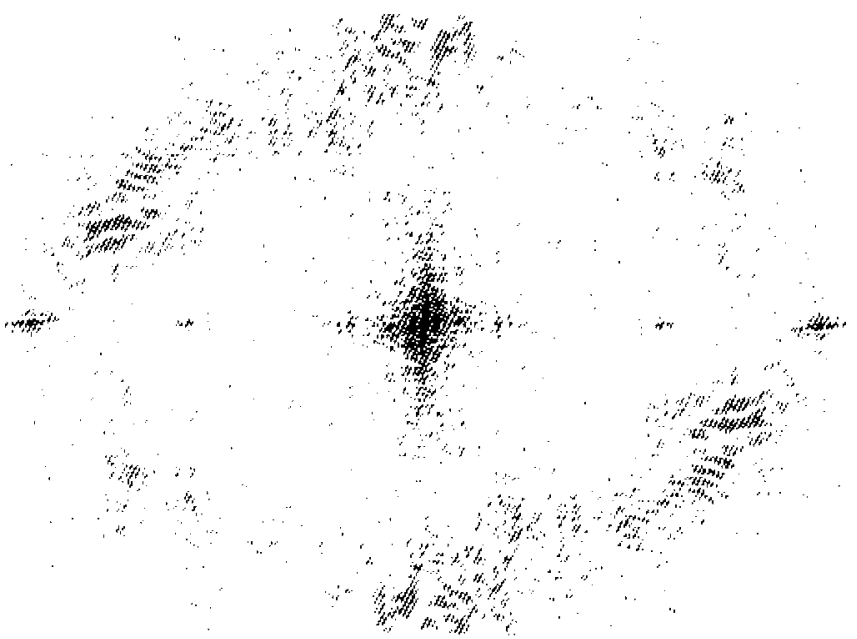

Fig. 10: Thresholded spectrum of fingerprint No.6

$$
\text { - constant thresholding }
$$

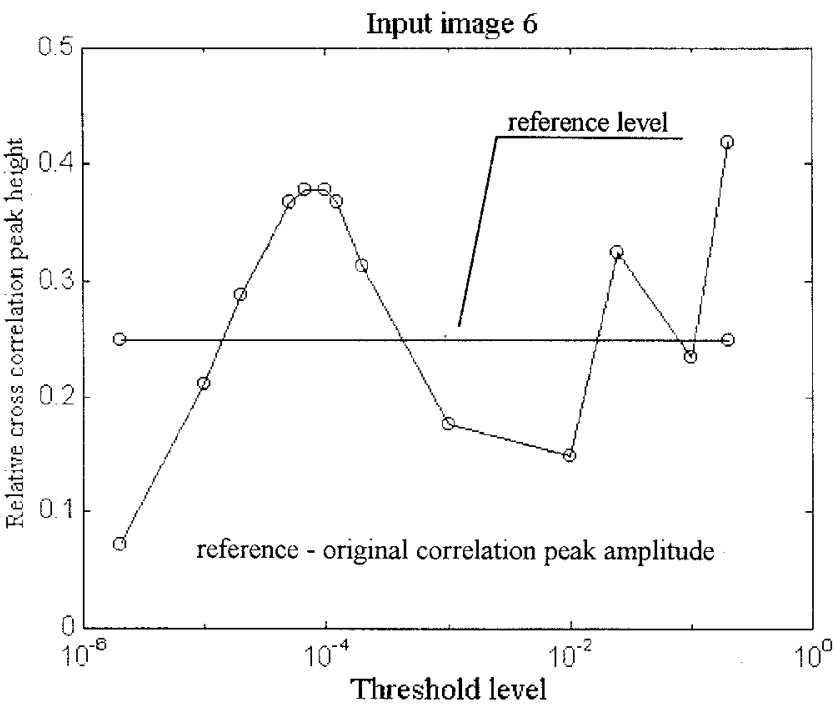

Fig. 11: Correlation peak vs. thresholding level - fingerprint No.6

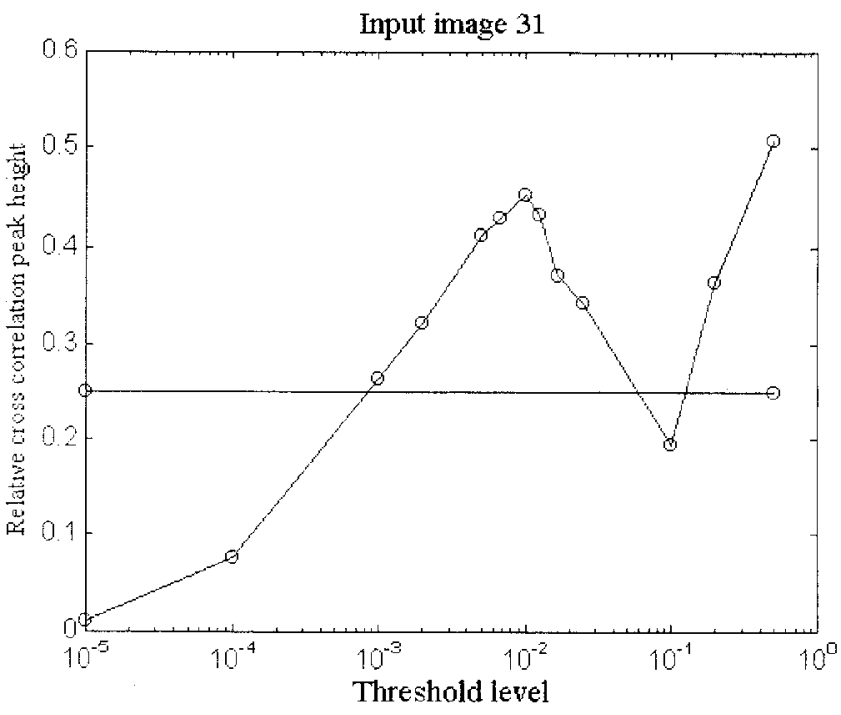

Fig. 12: Correlation peak vs. thresholding level - face No.31

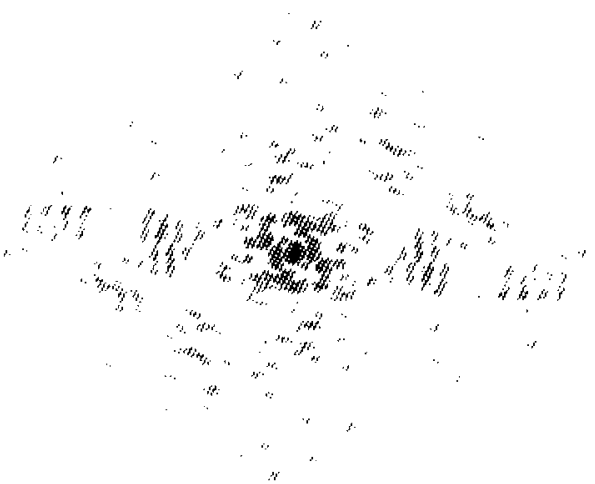

Fig. 13: Thresholded spectrum of face No 31

- constant thresholding 


\section{EXPONENTIAL THRESHOLDING IN THE SPECTRAL PLANE}

The other possible approach is based upon an implementation of variable thresholding in the spectral plane - an exponential function in our case. We have applied an exponential function crossing the peak value of the DC component and then this function has been shifted downwards. An example of a thresholded spectrum is shown in Fig.14. Again for both classes of object the shift has been optimized - see Fig. 15 for fingerprints and Fig. 16. for faces. The local correlation peak amplitude extremes are apparent on both diagrams.

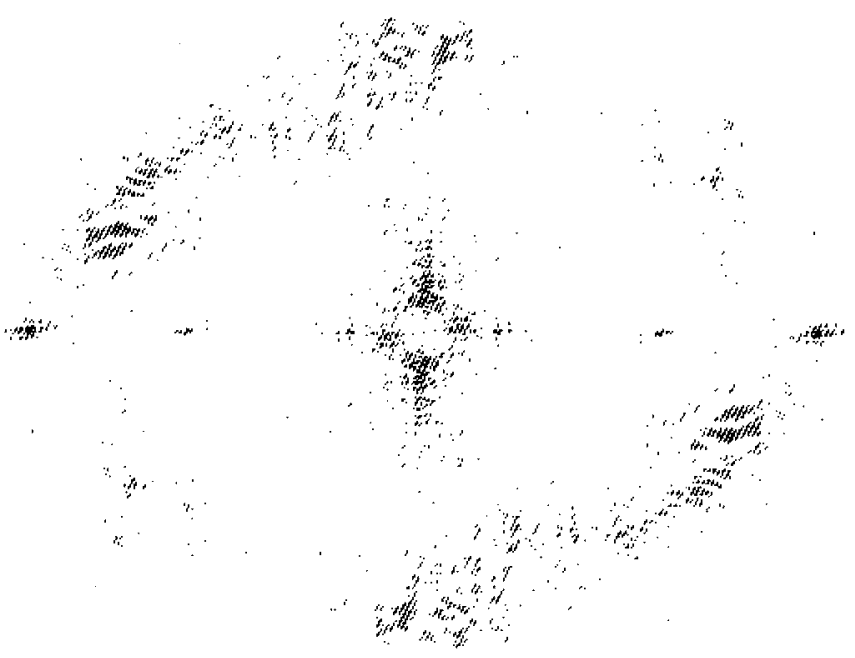

Fig. 14: Thresholded spectrum of fingerprint No.6 - exponential thresholding

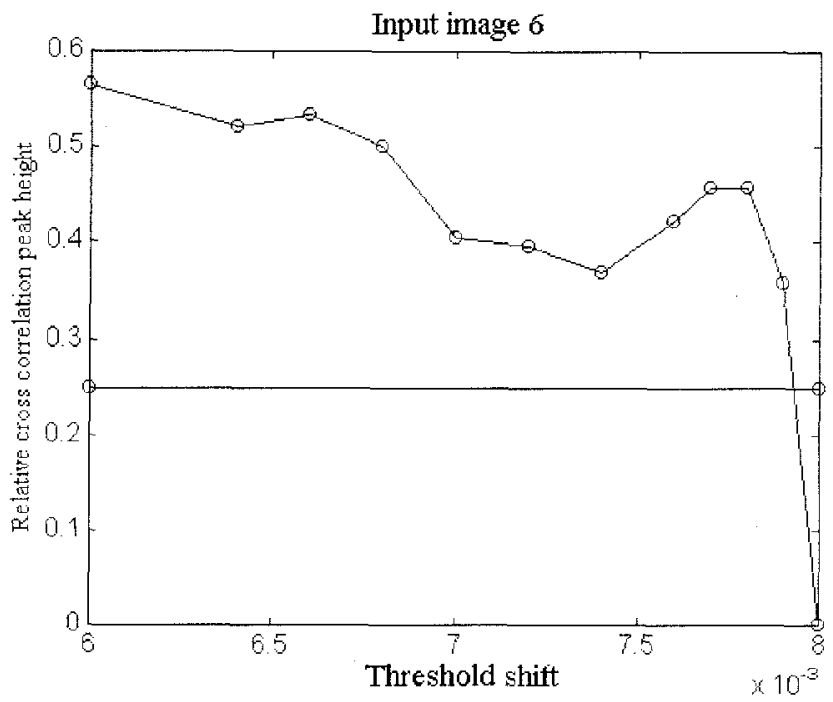

Fig. 15: Correlation peak vs. thresholding level - fingerprint No.6

$$
\text { - exponential thresholding }
$$

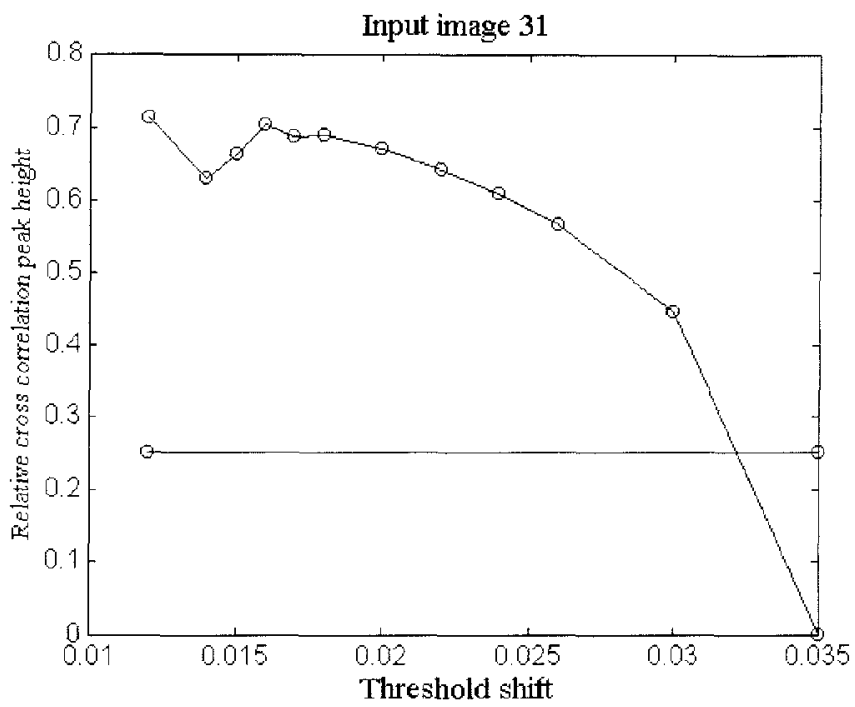

Fig. 16: Correlation peak vs. thresholding level - face No.31

$$
\text { - exponential thresholding }
$$

\section{CONCLUSION}

The presented paper has summarized some statistical testing of different JTC properties. For two particular classes of objects fingerprints and faces - the experimental dependencies of the correlation peaks on rotation and dilation have been measured. We can see in both cases the extent of the JTC's insensitivity while still correctly identifying inputs. The second part - thresholding of the spectrum seems to be a more unpredictable method. Both constant and exponential thresholding can improve the correlation peak amplitude but the actual level of thresholding is extremely object dependent. In the future we would like to evaluate a broader set of objects in order to find some common method of deriving the optimum threshold level and shape

This work has been supported by grants of the Czech Grant Agency No. 102/95/1157, HEIC 1/5227 and Forbairt IC/96/032

\section{REFERENCES}

[1] F.T.Yu, D.A.Gregory, Optical Pattern Recognition: Architectures and Techniques, Proc. of IEEE, 84, 5, May 1996, pp 733-752

[2] Klima M, Rott J., Gleeson D., McKenna-Lawlor S., Keating J, Model of 2D Optical Correlator for Fingerprint Identification., 30th Int. IEEE Conference on Security Technology, 1996, Lexington, USA

[3] Vanderiugt A., Optical Signal Processing, A Wiley Interscience Publication, John Wiley \& Sons, New York, 1992

[4] Lee J.N., Design Issues in Optical Processing, Cambridge University Press, 1995

[5] Volner R., Trunkvalter M., Interactive communications in CATV, Proceedings of Int. Conf. Electro95, Zilina, pp 252-256 